\title{
A hybrid de-noising method for mammogram images
}

\author{
Rashid Mehmood Gondal ${ }^{1}$, Saima Anwar Lashari ${ }^{2}$, Murtaja Ali Saare ${ }^{3}$, Sari Ali Sari ${ }^{4}$ \\ ${ }^{1}$ Department of Computer Science, the University of Lahore, Sargodha, Pakistan \\ ${ }^{2}$ College of Computing and Informatics, Saudi Electronic University, Dammam, Saudi Arabia \\ ${ }^{3}$ School of Computing, Universiti Utara Malaysia, Kadah, Malaysia \\ ${ }^{4}$ Faculty of Computer Science and Information Technology Universiti Tun Hussein, Johor, Malaysia
}

\begin{tabular}{|c|c|}
\hline Article Info & ABSTRACT \\
\hline Article history: & In general, mammogram images are contaminated with noise which directly \\
\hline Received May 17, 2020 & $\begin{array}{l}\text { ality. Several methods have been proposed to de-noise these } \\
\text { there is always a risk of losing valuable information. In }\end{array}$ \\
\hline Revised Sep 18, 2020 & order to overcome the loss of information, the present study proposed a \\
\hline Accepted Nov 11, 2020 & $\begin{array}{l}\text { Hybrid denoising method for mammogram images. The proposed hybrid } \\
\text { method works in two steps: Firstly, preprocessing with mathematical }\end{array}$ \\
\hline Keywords: & unsymmetrical trimmed median filter (GUTM) is applied to a de-noise \\
\hline $\begin{array}{l}\text { Breast cancer } \\
\text { Global unsymmetrical trimmed } \\
\text { median }\end{array}$ & $\begin{array}{l}\text { image. Experimental results prove that the proposed method works well for } \\
\text { mammogram images. Hence, the study provided an alternative method for } \\
\text { denoising mammogram images. }\end{array}$ \\
\hline
\end{tabular}

Hybrid denoising method

Mammogram images

This is an open access article under the CC BY-SA license.

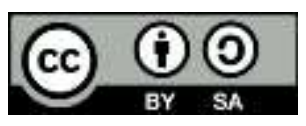

Corresponding Author:

Murtaja Ali Saare

School of Computing

Universiti Utara Malaysia

Sintok, 06010 Bukit Kayu Hitam, Kedah, Malaysia

Email: mmurtaja88@gmail.com

\section{INTRODUCTION}

Medical images carry valuable information that plays a vital role in the diagnosis and prognosis of diseases by taking significant images of the human body. It involves image classification, image segmentation [1], image matching, change in sequence, and feature extraction. Mostly the radiology department uses these medical images, in which diagnosis and prognosis of the disease are done are x-ray, computed tomography (CT), positron emission tomography (PET), magnetic resonance imaging (MRI), ultrasound, and digital mammogram images. A mammogram is one of the major types of medical images that are used for the early detection of breast cancer in women. As these images are contaminated with noise during their acquisition.

Different types of noises create an intensity in images which includes: gaussian noise, speckle noise, salt and pepper noise, amplifier noise, poison noise, and quantization. Image denoising is the technique used to avoid the problems of images such as signal distortion and unwanted noise. Image denoising takes a vital position in image processing. Image de-noising uses different methods such as median filtering, mean filtering, and winner filter to make the images clear and fine [2].

Basically, two types of models are used in image denoising: linear and nonlinear filtering. In early, liner filters such as mean and wiener filters were fundamental tools for image improvement. These techniques were easy to design [3] and implement. These filters have satisfying performance in many operations such as mean filter good for removing grain noise while wiener filter good for removing additive 
noise, both filters are also used for removing noise, however, sometimes it has poor results like linear filter mostly tend to blur the edge of pixel and didn't remove the Gaussian noise and mixed noise [4]. Therefore, the nonlinear denoising techniques having greater coefficients which show the signal and the noise coefficients are tried to reduce to none. These filtering techniques have various advantages and disadvantages [5-7]. Among the different filters, no one overcomes others with respect to computational cost, denoising, and enhancement of the resultant image. Therefore, each noise removal method can be improved further and still is an open research area.

Sawan [8] proposed an enhancement method for medical images by super-resolution. By using different filters such as total variations decomposition, stock filter, and linear interpolation. The stock filter works for edge enhancement and segmentation. In linear interpolation, unknown locations find out by known data values. Therefore, using the median, frost, and wiener a lot of noise is reduced. Kankariya and Gupta [9] proposed the arithmetic mean filtering technique that works automatically as the noise occurs. This technique work when the pixels in the image are founded corrupted, it removes the pixels and replaces that with estimated values. Jaiswal [10] proposed a new approached for the reduction of poison noise in digital images. For denoising median filter, wiener filter, thresholding techniques were applied. This technique works on image decomposition, using wavelet transform and then it applies hard thresholding and soft thresholding techniques for images denoising. This technique was used on 256x256 noisy images. So the best result was found by using the combination of the filtering method and threshold techniques.

Even though some existing linear and non-linear filters are good to de-noise and enhance medical images, however, not best for mammogram images [11]. The drawback behind is image may get blur and may lose some valuable information. To overcome the above-sited issue, a hybrid denoising method based on a global unsymmetrical trimmed median filter (GUTM) embedded with salt \& pepper noise is proposed in this study. Based on the research background and the related issues, the objective of this research has been formulated as:

a) To propose a hybrid denoising method based on a global unsymmetrical trimmed median filter (GUTM) for denoising mammogram images.

b) To evaluate hybrid denoising method by using mean square error (MSE) [12], peak-signal-to-noise ratio (PSNR) [13], and structural similarity index metric (SSIM) [14].

c) To validate the performance of the proposed method with existing techniques namely mean, median and winner filter. Performance evaluations of proposed filters are validated with mean, median and winner filter the results are in favor of the proposed method with respect to PSNR, MSE, and SSIM.

\section{RESEARCH METHODOLOGY}

The methodology used in the present study composed of eight steps: Firstly a grayscale mammogram image is loaded in mat-lab and assumed as the original image after that image is passed through some pre-processing such as contrast enhancement and intensity change [15]. When the preprocessing phase is completed mathematical morphological function erosion is applied to an image. Later on, salt \& pepper noise is embedded in the above image and the image becomes degrade and noisy. For denoising images, select a processing window size for the proposed global unsymmetrical trimmed median filter (GUTM) and the proposed method. The quality of the processed image is evaluated using peak signal to noise ratio (PSNR), mean square error (MSE) structure similarity index matrix (SSIM), and results are compared with existing median, mean, and winner filter technique. Figure 1 illustrates the research design.

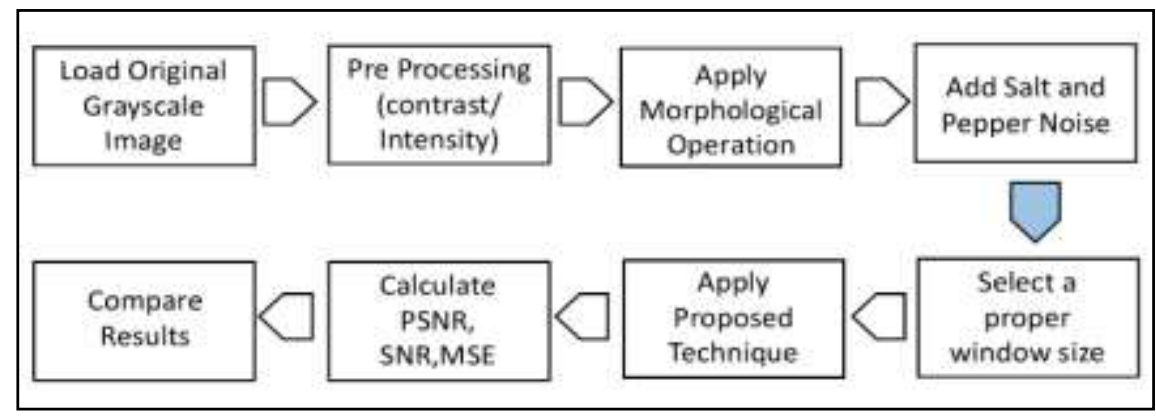

Figure 1. Research design 


\section{DATA COLLECTION}

Images for this work are taken from mammogram images analysis society (MIAS). MIAS is a UK research group organization that is interested in mammograms and has generated a database of digital mammogram images. These images are available free of cost on the MIAS website. MIA's dataset contains 322 images collected from 161 patients of both left and right breasts. All the images are gray level and the format is a portable gray map (PGM) [16]. Based on severity, images are classified into three categories described in Table 1.

Table 1. Image description

\begin{tabular}{ccc}
\hline S. No. & MIAS & Description \\
\hline 1 & Normal & 207 \\
2 & Benign & 61 \\
3 & Malignant & 54 \\
\hline
\end{tabular}

\section{MATHEMATICAL MORPHOLOGY}

Mathematical morphology is a set of the theoretical method of image analysis and provides a characterization of the geometrical structure of an image [17]. Basic operational steps of mathematical morphology are erosion and dilation where all other operations such as opening, closing are derived from these two operations. Combination, opening, and closing operations are used to form a traditional morphological filter. Suppose the discrete input signal $\mathrm{f}(\mathrm{n})$ is defined as $\mathrm{F}=\{0,1, \ldots \ldots \ldots, \mathrm{X}\}$ and the structural element $\mathrm{g}(\mathrm{n})$ is defined as $\mathrm{G}=\{0,1, \ldots ., \mathrm{Y}\} \mathrm{Y} \geq \mathrm{X}$, then the erosion and dilation operations are defined as follows [18]:

Dilation:

$$
(\mathrm{fOg})(\mathrm{y})=\max \{\mathrm{f}(\mathrm{y}-\mathrm{x})+\mathrm{g}(\mathrm{x})\}
$$

Erosion:

$$
\begin{aligned}
& (f O g)(y)=\min \{f(y-x)+g(x)\} \\
& \text { Where } y=\{0,1, \ldots, Y-1\} \text { and } x=\{0,1, \ldots, X-1\} .
\end{aligned}
$$

\section{PROPOSED TECHNIQUE GUTM (GLOBAL UNSYMMETRICAL TRIMMED MEDIAN FILTER)}

Global unsymmetrical trimmed median filter using salt and pepper noise is illustrated in Figure 2, where the grayscale image is loaded in and $3 * 3$-kernel size is selected. Processing pixel P (i, j) is analyzed whether it is noisy or noise-free. However, if the processing pixel is in between the minimum and maximum value of a grayscale image its mean pixel is noise-free and left unchanged. However, if the pixel takes a minimum and maximum gray level value its mean pixel is corrupted with salt or pepper noise and processed according to the proposed method. Figure 2 illustrates the proposed technique.

Proposed Technique:

Step 1: Select a 2D window having size $3 * 3$ Assuming that the processing pixel $P(i, j)$ is lies at the center of the pixel.

Step 2: Check for the processing pixel is corrupted or uncorrupted.

Step 3: If $0<P(i, j)<255$ mean processing pixel lie between minimum and maximum so it is uncorrupted and left unchanged.

Step 4: If the processing pixel $P(i, j)=0$ or $P(i, j)=255$ then it is taken as noisy and processed by the next instruction.

Step 5: Arrange the pixel value in the current processing window in ascending order.

Step 6: Check the No of a non-noisy pixel in the window and store total no in a variable called ' $N$ '

Step 7: Based on the value of ' $N$ ' there are the following possible cases.

Step 8: If the value of ' $\mathrm{N}$ ' is zero its mean window did not contain and noise-free pixel so there are three possible cases or go to step 9 .

Case I: if the window contains all the element ' 0 ' pepper noise then replace the noisy pixel $\mathrm{P}(\mathrm{i}, \mathrm{j})$ with salt noise (e.g. 255) trimmed Global Unsymmetrical Median filter of the entire image.

Case II: if the window contains all the element ' 255 ' salt noise then replace the noisy pixel $\mathrm{P}(\mathrm{i}, \mathrm{j})$ with pepper noise (e.g. 0) trimmed Global Unsymmetrical Median filter of the entire image. 
Case III: if the window contains all the elements ' 0 ' \& ' $255^{\prime}$ ' or $\mathrm{N}<=4$ then replace the noisy pixel $\mathrm{P}(\mathrm{i}, \mathrm{j})$ with the median of the selected window.

Case IV: If the value of ' $\mathrm{N}$ ' is greater or equal to five then take an unsymmetrical trimmed median of the selected window and replace the processing pixel.

Step 9: Move to the next pixel and repeat from step 1 to 10 for a remaining pixel in the window.

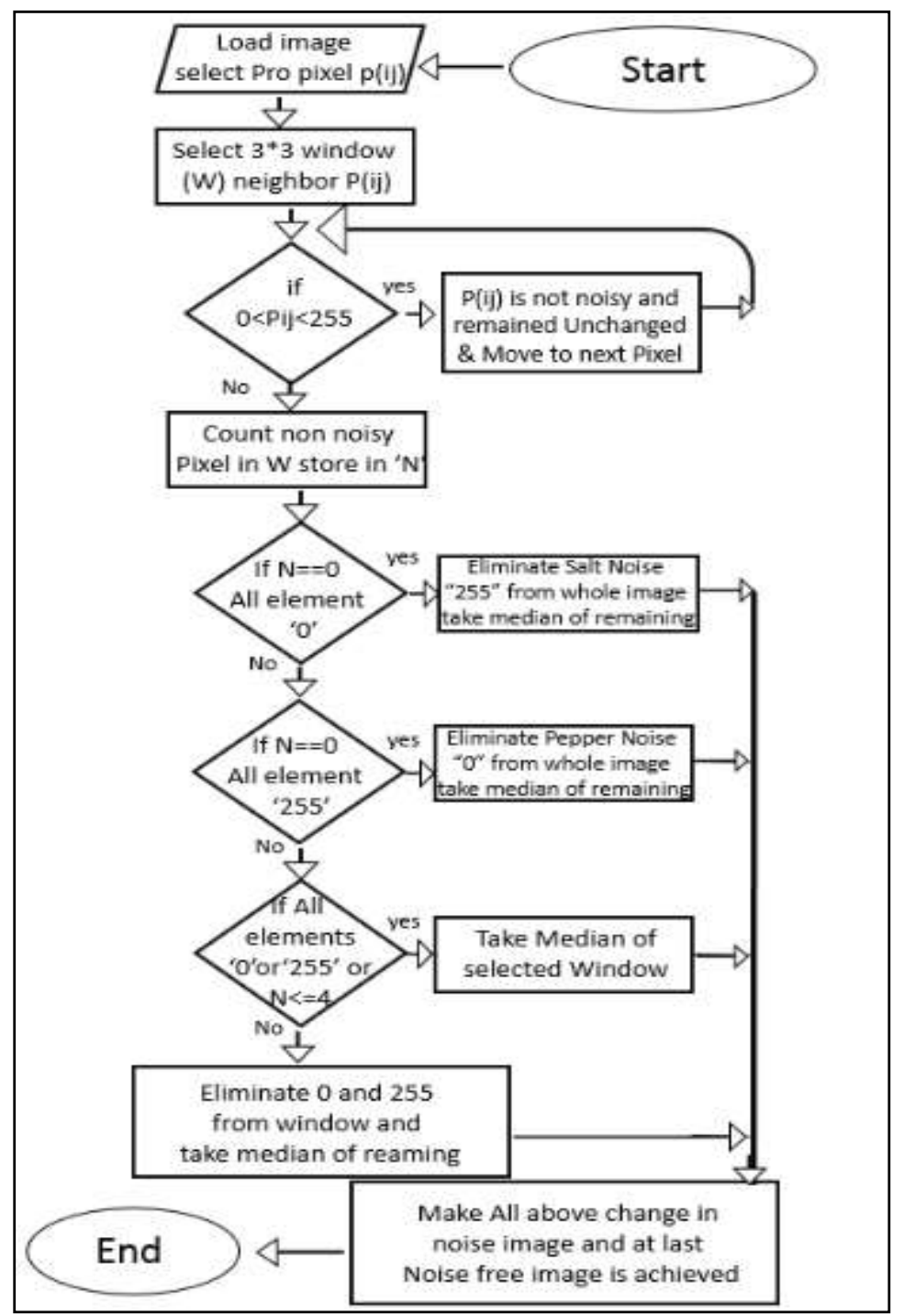

Figure 2. Flow chart of the proposed technique

\section{ILLUSTRATION OF PROPOSED METHODOLOGY}

In this section, two different matrices will be discussed. The bigger matrix shows the image segment during the experiment from the original image, and a $3 * 3$ matrix shows the processing window. Element circled on the left side are taken as processing pixel $P(i, j)$ and on the right side, the matrix is taken as a restored pixel. The square box refers to the current processing window. If processed Pixel is between $0 \& 255$ so it's mean not noisy and remains unchanged. In this case P $(\mathrm{i}, \mathrm{j})$ hold the value 5 which is not equal to 0 or 255 and is considered non-noisy, and is kept unchanged. Figure 3 illustrates the process to find whether the pixel is noisy or not.

\section{Case I:}

The $\mathrm{P}(\mathrm{i}, \mathrm{j})$ is noisy ' 0 ' and all its neighbor in the selected window are ' 0 ' as all the pixel in the window are zero (pepper noise) so in this case, check the whole image and remove all salt noise from the whole image and take the median of reaming pixels in entire image and replace with current pixel. Figure 4 illustrates case I. 


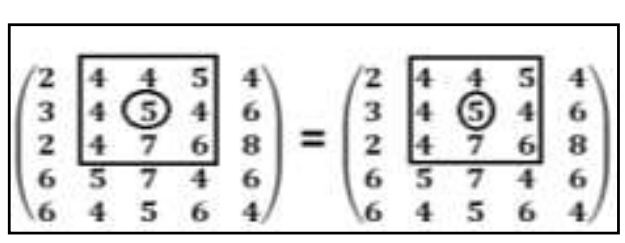

Figure 3. Illustration to find the noisy pixel

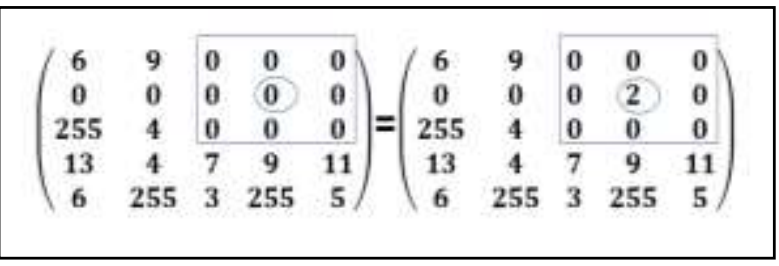

Figure 4. Illustration of the case I

In Figure 4 selected window contain all the pixel pepper noise ' 0 ' so take all the element of image and change 2D array into 1D (690000000025540001347911 625532555) now trim the salt noise (e.g. 255) from Array and arrange reaming in ascending order. (00000000 0003445667991113 ). Find the median of $1 \mathrm{D}$ array as the center elements of sorted array are 0 and 3 so median is $(0+3) / 2=1.5$ almost 2. Now replace the processing pixel with 2 as in Figure 4 on right side.

\section{Case II:}

The $\mathrm{P}(\mathrm{i}, \mathrm{j})$ is noisy ' 255 ' and all it's neighbor in a selected window are ' 255 ' as all the pixel in the window are 255 (salt noise) so in this case, check the whole image and remove all pepper noise from the whole image and take the median of reaming pixels in entire image and replace with processing pixel. Figure 5 illustrates case II.

$\left(\begin{array}{ccccc}255 & 255 & 255 & 3 & 9 \\ 255 & 255 & 255 & 7 & 2 \\ 255 & 255 & 255 & 0 & 0 \\ 0 & 4 & 4 & 9 & 255 \\ 6 & 2 & 3 & 255 & 5\end{array}\right)=\left(\begin{array}{ccccc}255 & 255 & 255 & 3 & 0 \\ 255 & 132 & 255 & 7 & 2 \\ 255 & 255 & 255 & 0 & 0 \\ 0 & 4 & 0 & 9 & 255 \\ 6 & 0 & 3 & 255 & 5\end{array}\right)$

Figure 5. Illustration of case II

In Figure 5 selected window contain all the pixel salt noise ' 255 ' so take all the elements of the image and change the 2D array into 1D (255 255255392552552557225525525500 ) now trim the pepper noise (e.g. 0) from Array and arrange to remain in ascending order. (2 2334456799255255255 255255255255255255255255 ). Find the median of the 1D array as the central elements of the sorted array are 9 and 255 so the median is $(9+255) / 2=132$. Now replace the processing pixel with 132 as in Figure 5 on the right side.

\section{Case III:}

The $\mathrm{P}(\mathrm{i}, \mathrm{j})$ is noisy ' 0 ' or ' 255 ', and it's neighbor in a selected window are noisy and non-noisy. Now check and count non-noisy pixel if these are less or equal to 4 arrange all the elements in a window in ascending order and find the median and replace with processing pixel. Figure 6 illustrate case III.

$\left(\begin{array}{ccccc}3 & 9 & 3 & 255 & 3 \\ 5 & 3 & 0 & 3 & 4 \\ 3 & 7 & 4 & 255 & 3 \\ 8 & 255 & 3 & 0 & 255 \\ 3 & 3 & 0 & 4 & 0\end{array}\right)=\left(\begin{array}{ccccc}3 & 9 & 3 & 255 & 3 \\ 5 & 3 & 0 & 3 & 4 \\ 3 & 7 & 4 & 255 & 3 \\ 8 & 255 & 3 & 3 & 255 \\ 3 & 3 & 0 & 4 & 0\end{array}\right)$

Figure 6. Illustration of case III

In the Figure 6, processing pixel $\mathrm{P}(\mathrm{i}, \mathrm{j})$ is 0 selected window contain pixel noisy and non-noisy. Now count a number of non-noisy pixels and store in variable ' $N$ '. As in this situation ' $N$ ' is equal to 4. Change the element of the selected window from 2D array to 1D (4 255330255040 ) Arrange elements in 
ascending order. ( 0003344255 255). Find the median of the 1D array as the central elements of a sorted array is 3 so the median is 3 . Now replace the processing pixel ' 0 ' with ' 3 ' as in Figure 6 on the right side.

\section{Case IV:}

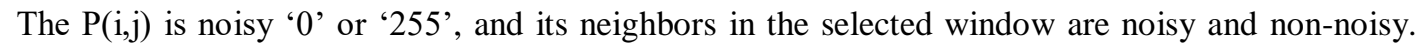
Now check and count non-noisy pixel if these are greater or equal to 5 arrange all the elements in a window in ascending order trim ' 0 ' and ' 255 ' find the median of remaining to replace with processing pixel. Figure 7 illustrates case IV.

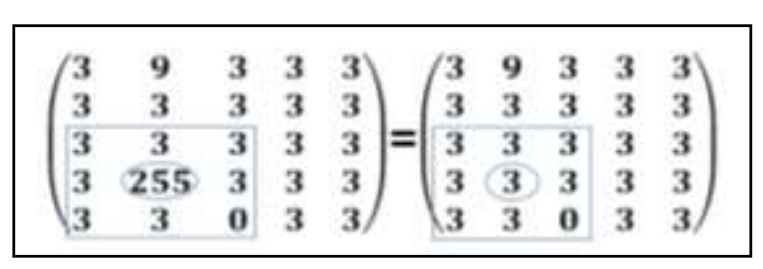

Figure 7. Illustration of case IV

In the Figure 7 processing pixel $\mathrm{P}(\mathrm{i}, \mathrm{j})$ is 255 selected windows contain pixel noisy and non-noisy. Now count the number of non-noisy pixels and store in variable ' $N$ '. As in this situation ' $N$ ' is equal to 7 greater than 5. Change the element of the selected window from 2D array to 1D (3333 255333 0) Arrange elements in ascending order. ( 03333333 255). Trim ' 0 ' and ' 255 ' from the array and find the median of reaming array $\left(\begin{array}{llll}3 & 333333\end{array}\right)$ as the central elements of a sorted array is 3 so median is 3 . Now replace the processing pixel ' 255 ' with ' 3 ' as in Figure 7 on the right side.

\section{RESULTS AND DISCUSSION}

This section describes the validation scenarios of the proposed methodology with mathematical morphology (MM) and in the absence of MM. Experiments were conducted on different normal, benign, and malignant mammogram images. Images were embedded with salt and pepper noise. Experiments were performed and results are compared with other existing denoising techniques such as; mean filter, median filter, and Wiener filter. Results indicate that the proposed GUTM technique exhibits better quantitative measurements in the form of PSNR, MSE. SSIM [19].

A. Peak Signal-to-Noise Ratio (PSNR)

$$
P S N R=10 \log _{10}\left(\frac{M a x_{I}^{2}}{M S E}\right)=20 \log _{10}\left(\frac{\text { MaxI }}{M S E}\right) \text {--------[12] }
$$

B. Measurement of MSE

The MSE is defined as:

$$
M S E=\sum_{x=y=1}^{N}[f(x, y)-F(x, y)]^{2} / N^{2}
$$

C. Structural Similarity Index Metric (SSIM)

The SSIM is computes as:

$$
\operatorname{SSIM}(a, b)=\frac{(2 \times \bar{a} \bar{b}+c 1)\left(2 \times \sigma_{a b}+c 2\right)}{\left(\sigma_{a}^{2}+\sigma_{b}^{2}+c 2\right) \times\left((\bar{a})^{2}+(\bar{b})^{2}+c 1\right)}
$$

Where

$\bar{a}:$ is the average of $a, \bar{b}:$ is the average of $b, \sigma_{a b}:$ represent the standard deviation of $a$ and $b$.

Comparison of different techniques such as mean filter, winner filter, median filter, median filter with mathematical morphology, global unsymmetrical trimmed median filter, and proposed hybrid GUTM is shown in the Table 2. Results show that the proposed technique works better for mammogram images having PSNR 52.31 and MSE 0.37 and SSIM 0.99. Table 2 shows a comparison of the proposed technique with existing. Figures 8-10 shows PSNR, MSE, and SSIM for mammogram image. Figures 11-13 illustrate the graphical representation of PSNR, MSE, SSIM values for several mammogram images respectively. 
Table 2. Shows the PSNR, MSE, SSIM value for normal, benign, and malignant images

\begin{tabular}{|c|c|c|c|c|c|c|c|c|}
\hline Test Type & $\begin{array}{l}\text { Severity of } \\
\text { Image }\end{array}$ & $\begin{array}{l}\text { Image } \\
\text { Name }\end{array}$ & $\begin{array}{l}\text { Mean } \\
\text { Filter }\end{array}$ & $\begin{array}{c}\text { Winner } \\
\text { Filter }\end{array}$ & $\begin{array}{l}\text { Median } \\
\text { Filter }\end{array}$ & $\begin{array}{l}\text { Median Filter } \\
\text { With MM }\end{array}$ & GUTM & $\begin{array}{c}\text { GUTM with } \\
\text { MM }\end{array}$ \\
\hline \multirow{3}{*}{ PSNR } & Normal & mdb322 & 28.78 & 20.99 & 36.2 & 39.58 & 45.32 & 52.31 \\
\hline & Benign & mdb188 & 28.28 & 20.52 & 38.31 & 39.2 & 47.92 & 49.65 \\
\hline & Malignant & $\operatorname{mdb} 216$ & 28.62 & 20.95 & 35.29 & 37.62 & 43.29 & 48.44 \\
\hline \multirow{3}{*}{ MSE } & Normal & mdb322 & 86.05 & 517.25 & 9.32 & 7.14 & 0.98 & 0.37 \\
\hline & Benign & Mdb005 & 76.91 & 483.8 & 3.45 & 8.64 & 1.52 & 0.53 \\
\hline & Malignant & $\mathrm{mdb} 256$ & 83.14 & 495.08 & 16.01 & 10.48 & 1.24 & 0.5 \\
\hline \multirow{3}{*}{ SSIM } & Normal & $\mathrm{mdb} 018$ & 0.19 & 0.12 & 0.89 & 0.94 & 0.98 & 0.99 \\
\hline & Benign & $\mathrm{mdb} 001$ & 0.25 & 0.16 & 0.95 & 0.95 & 0.99 & 0.99 \\
\hline & Malignant & $\operatorname{mdb} 216$ & 0.36 & 0.23 & 0.89 & 0.94 & 0.98 & 0.99 \\
\hline
\end{tabular}

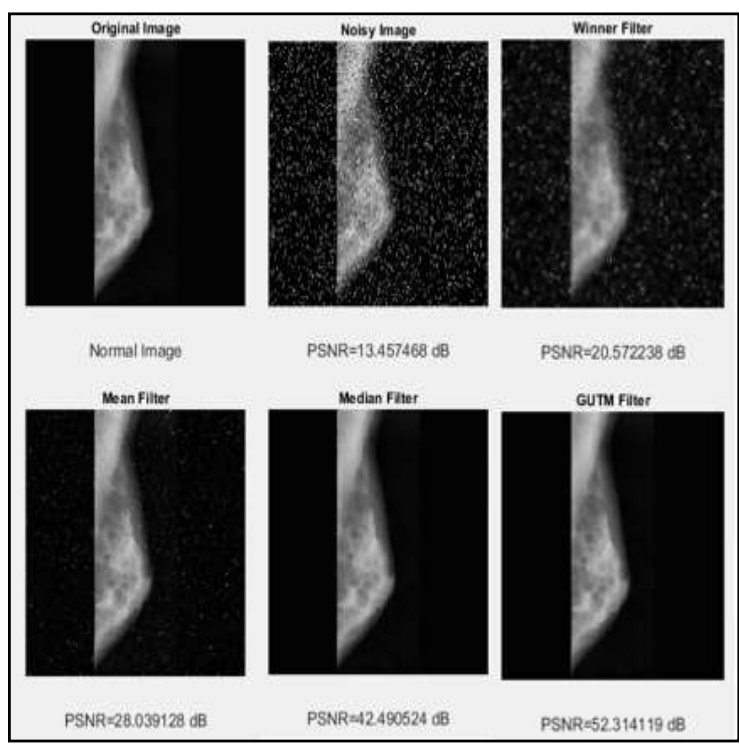

Figure 8. Comparison of PSNR for normal image

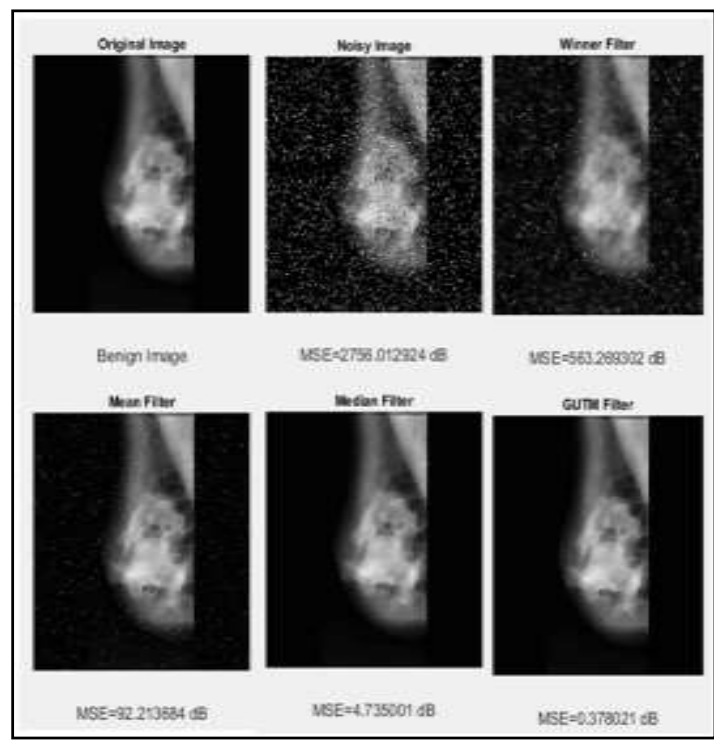

Figure 9. Comparison of MSE for benign image

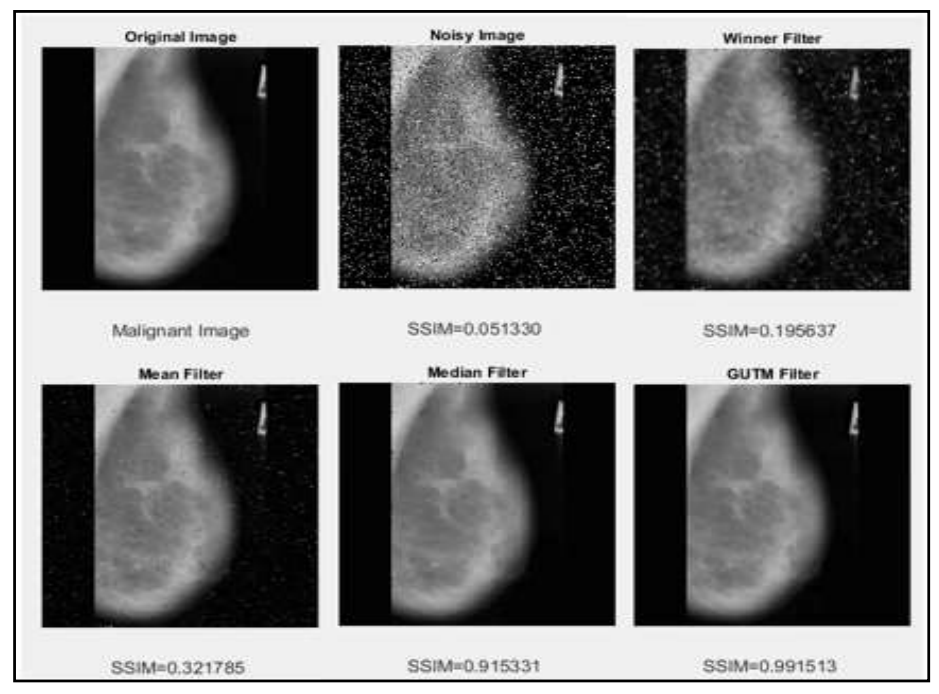

Figure 10. Comparison of SSIM for malignant image 


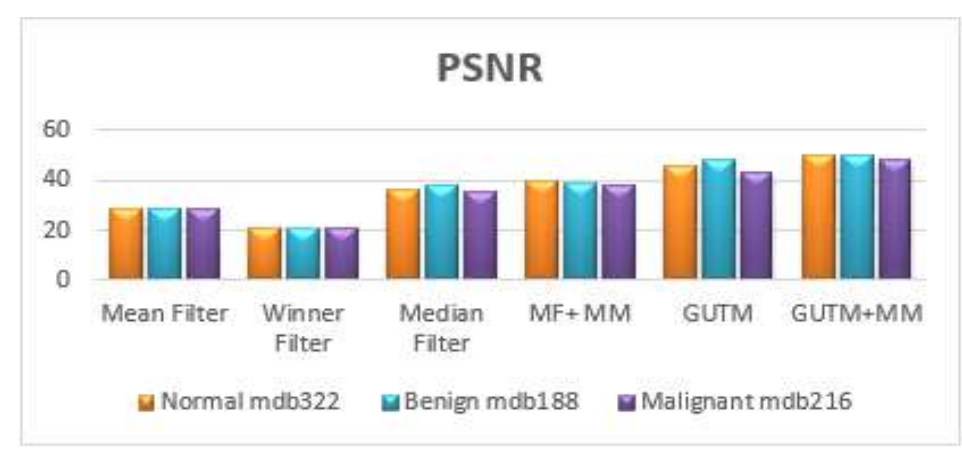

Figure 11. Graphical representation of PSNR for normal, benign, and malignant image

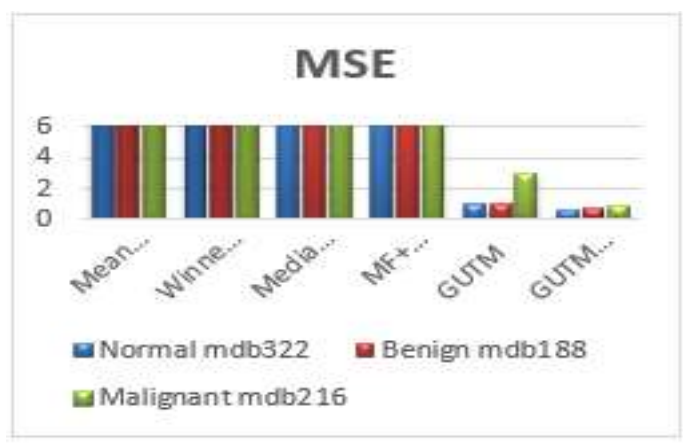

Figure 12. Graphical representation of MSE for normal, benign, and malignant image

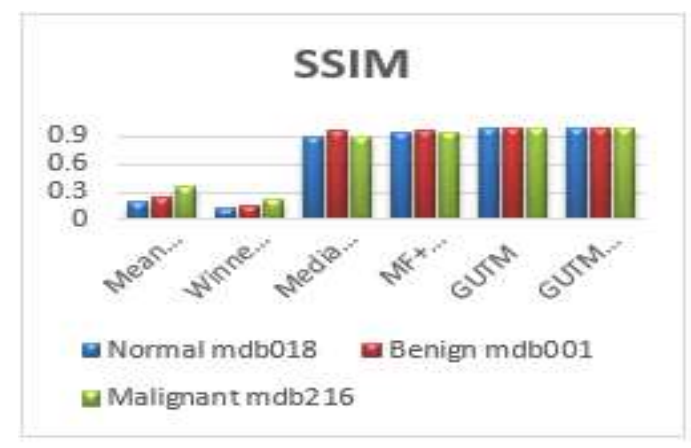

Figure 13. Graphical representation of SSIM for normal, benign, and malignant image

\section{COMPARISON OF EXISTING TECHNIQUE WITH PROPOSED HYBRID DENOISING METHOD}

In this section, a comparison of different techniques namely adaptive median filter, mean filter, hybrid median filter, moving average filter and gaussian low pass filter is performed with the proposed hybrid GUTM method. Results show that the proposed technique works better for mammogram images having PSNR 52.31 and MSE 0.37. Table 3 shows the comparison of the proposed technique with related work.

Table 3. Comparison of PSNR and MSE value for mammogram image

\begin{tabular}{|c|c|c|c|c|}
\hline Technique & Year & Noise Type & PSNR & MSE \\
\hline Db3 Wavelet Transform [22] & 2014 & Gaussain Noise & 48.79 & 0.85 \\
\hline HAAR Wavelet Transform [22] & 2014 & Gaussain Noise & 48.30 & 0.96 \\
\hline Median FILter And CLAHE [23] & 2015 & Mix Noise & 50.67 & 0.90 \\
\hline $\begin{array}{c}\text { Fast Discrete Curvelet Transform via UneqiSpaced Fast Fourier } \\
\text { Transform [24] }\end{array}$ & 2017 & Several noise & 39.42 & 7.43 \\
\hline Multiple description Gaussian noise channel [25] & 2017 & Gaussian Noise & 37.87 & 10.63 \\
\hline Moving Average Filter [26] & 2018 & Salt \& Pepper & 35 & 3.4 \\
\hline Gaussian Low pass filter [27] & 2018 & Salt \& Pepper & 38 & 1.3 \\
\hline Tristate filter (TSF) [24] & 2019 & Salt \& Pepper & 44.71 & 2.4 \\
\hline Hybrid Denoising Method based on GUTM (Proposed Method) & 2020 & Salt \& Pepper and Gausain & 52.31 & 0.37 \\
\hline
\end{tabular}

\section{CONCLUSION}

In this work, a hybrid denoising method is proposed for mammogram images. Mammogram contains useful information about the diagnosis and prognosis of breast cancer in women. However, noise in mammogram often disturb image important information and make it difficult for the radiologist to diagnosis. The proposed hybrid method is used to de-noise the image and to enhance the image quality by keeping image important information unchanged. Different experimental setups were designed to obtain PSNR, MSE, and SSIM. Later, validated with existing denoising techniques namely mean falter, wiener filter, and median filter. Results show that the proposed method performs well for denoising mammogram images. 


\section{REFERENCES}

[1] S. A. Sari and K. M. Mohamad, "A Review of Graph Theoretic and Weightage Techniques in File Carving," in Journal of Physics: Conference Series, 2020, vol. 1529, no. 5, p. 52011.

[2] L. Shao, R. Yan, X. Li, and Y. Liu, "From heuristic optimization to dictionary learning: A review and comprehensive comparison of image denoising algorithms," IEEE Trans. Cybern., vol. 44, no. 7, pp. 1001-1013, 2013.

[3] M. A. Al-Shareeda, M. Anbar, S. Manickam and A. A. Yassin, 'VPPCS: VANET-Based Privacy-Preserving Communication Scheme,' in IEEE Access, doi: 10.1109/ACCESS.2020.3017018.

[4] D. Semrau, R. I. Killey, and P. Bayvel, "The Gaussian noise model in the presence of inter-channel stimulated Raman scattering," J. Light. Technol., vol. 36, no. 14, pp. 3046-3055, 2018.

[5] K. Akila, L. S. Jayashree, and A. Vasuki, "Mammographic image enhancement using indirect contrast enhancement techniques-a comparative study," Procedia Comput. Sci., vol. 47, pp. 255-261, 2015.

[6] M. A. Al-Shareeda, M. Anbar, I. H. Hasbullah, S. Manickam, and S. M. Hanshi, "Efficient conditional privacy preservation with mutual authentication in vehicular ad hoc networks," IEEE Access, 2020.

[7] M. Al Shareeda, A. Khalil, and W. Fahs, "Realistic heterogeneous genetic-based RSU placement solution for V2I networks.," Int. Arab J. Inf. Technol., vol. 16, no. 3A, pp. 540-547, 2019.

[8] A. S. Sawan, S. S. Kamdi, D. M. Khatri, D. S. Urhekar, and C. D. Bohra, "Novel filter designing for enhancement of medical images using super-resolution," in 2017 International Conference on Signal Processing and Communication (ICSPC), pp. 253-257, 2017.

[9] C. Kankariya and S. Gupta, "An Efficient Algorithm for Removal of Salt and Pepper Noise from Images," Int. J. Comput. Appl., vol. 118, no. 18, 2015.

[10] A. Jaiswal, J. P. Upadhyay, R. Mohan, and S. P. Bohre, "A Novel Approach for Reduction of Poisson Noise in Digital Images," Int. J. Eng. Res. Appl., vol. 3, no. 5, pp. 1275-1279, 2013.

[11] X. Guo, J. Jiang, L. Tan, and S. Du, "Improved adaptive recursive even mirror Fourier nonlinear filter for nonlinear active noise control," Appl. Acoust., vol. 146, pp. 310-319, 2019.

[12] Y. Zhang et al., "A poisson-gaussian denoising dataset with real fluorescence microscopy images," in Proceedings of the IEEE Conference on Computer Vision and Pattern Recognition, 2019, pp. 11710-11718.

[13] K.-S. Lu, A. Ortega, D. Mukherjee, and Y. Chen, "Perceptually inspired weighted MSE optimization using irregularity-aware graph Fourier transform," arXiv Prepr. arXiv2002.08558, 2020.

[14] J. Wang, N. Zheng, B. Chen, and J. C. Principe, "Associations among image assessments as cost functions in linear decomposition: MSE, SSIM, and Correlation Coefficient," arXiv Prepr. arXiv1708.01541, 2017.

[15] G. Bicchierai et al., "Role of contrast-enhanced spectral mammography in the post biopsy management of B3 lesions: preliminary results," Tumori J., vol. 105, no. 5, pp. 378-387, 2019.

[16] R. Vijayarajeswari, P. Parthasarathy, S. Vivekanandan, and A. A. Basha, "Classification of mammogram for early detection of breast cancer using SVM classifier and Hough transform,” Measurement, vol. 146, pp. 800-805, 2019.

[17] T. Xu, G. Wang, H. Wang, T. Yuan, and Z. Zhong, "Gap measurement of point machine using adaptive wavelet threshold and mathematical morphology," Sensors, vol. 16, no. 12, p. 2006, 2016.

[18] X. Zhang, W. Qi, Y. Cen, H. Lin, and N. Wang, "Denoising vegetation spectra by combining mathematicalmorphology and wavelet-transform-based filters," J. Appl. Remote Sens., vol. 13, no. 1, p. 16503, 2019.

[19] E. Shimeles, G. Abebe, and A. Regasa, "Comparing the Performance of Various Speckle Reduction Filters on Ultrasound Kidney Stone Images.” Haramaya University, 2017.

[20] R. Kochher, G. Marken, and A. Oberoi, "Mammogram Restoration under Impulsive Noises using Peer GroupFuzzy Non-Linear Diffusion Filter."

[21] S. Kannan, N. P. Subiramaniyam, A. T. Rajamanickam, and A. Balamurugan, "Performance comparison of noise reduction in mammogram images," Image (IN)., vol. 1, no. 3x3, p. 5x5, 2016.

[22] S. A. Lashari, R. Ibrahim, and N. Senan, "De-noising analysis of mammogram images in the wavelet domain using hard and soft thresholding," in 2014 th World Congress on Information and Communication Technologies (WICT 2014), pp. 353-357, 2014.

[23] A. Makandar and B. Halalli, "Breast cancer image enhancement using median filter and CLAHE," Int. J. Sci. Eng. Res., vol. 6, no. 4, pp. 462-465, 2015.

[24] B. Senthilkumar, R. Gowrishankar, M. Vaishnavi, and S. Gokila, "Combination of noise removal and contrast enhancement methods for the preprocessing of mammogram images-towards the detection of breast cancer," Biosci. J., vol. 33, no. 6, 2017.

[25] J. Østergaard, Y. Kochman, and R. Zamir, "An Asymmetric Difference Multiple Description Gaussian Noise Channel," in 2017 Data Compression Conference (DCC), pp. 360-369, 2017.

[26] Y. Gherghout, Y. Tlili, and L. Souici, "Classification of breast mass in mammography using anisotropic diffusion filter by selecting and aggregating morphological and textural features," Evol. Syst., pp. 1-30, 2019.

[27] X. Duan et al., "A multiscale contrast enhancement for mammogram using dynamic unsharp masking in Laplacian pyramid," IEEE Trans. Radiat. Plasma Med. Sci., vol. 3, no. 5, pp. 557-564, 2018. 